\title{
Micromorfología de la epidermis foliar de cultivares de arroz Venezolano (Poaceae) asociado con el daño mecánico de sogata Tagosodes orizicolus (Homoptera: Delphacidae)
}

\author{
Rosalía Velásquez-Salazar ${ }^{1} \&$ Diego Diamont ${ }^{2}$ \\ 1. Instituto de Genética, Facultad de Agronomía-UCV, Maracay; rvelasquezsalazar@gmail.com \\ 2. Instituto Nacional de Investigaciones Agrícolas, Centro Nacional de Investigaciones Agropecuarias, Maracay (INIA- \\ CENIAP); diegodiamont@yahoo.com
}

Recibido 07-VI-2013. Corregido 19-XII-2013. Aceptado 20-I-2014.

\begin{abstract}
Micromorphology of leaf epidermis of some Venezuelan rice cultivars (Poaceae) associated with the mechanical damage of Sogata T. orizicolus (Homoptera: Delphacidae). Rice cultivars are affected directly and indirectly by the insect sogata. The mechanical damage or direct loss, is produced after feeding and ovoposition on the young leaves tissues, while the indirect damage is produced after the transmission of the Rice hoja blanca virus. We studied the morpho-anatomic structures associated with the resistance of the mechanical damage produced by the insect, in six rice cultivars, including controls for resistance and susceptibility (Makalioka and Bluebonnet 50), during August 2011, in Fundacion Danac, Venezuela. Samples were taken from leaf 3, where cuticle thickness, presence of macrohair, microhair and silica bodies in the second third of the leaf was evaluated. A significant difference to thickness of the cuticle, the presence of microhair in the leaves, and presence of silica bodies was observed among cultivars, determining a significant correlation among the number of macrohair and microhair in the adaxial leaf blade with the presence of silica bodies, and thickness of the cuticle and number of posed insects. Thickness of the cuticle and presence of silica bodies in the intercostals space of microhair and macrohair showed to be the variables most related to mechanical damage and resistance mechanism. Rev. Biol. Trop. 62 (2): 819-827. Epub 2014 June 01.
\end{abstract}

Key words: rice, sogata, anatomy, resistance, resistance mechanism.

El arroz (Oryza sativa L.) además de ser considerado el segundo cereal de mayor producción y consumo a nivel mundial, después del trigo, constituye la fuente alimenticia de mayor importancia por la superficie cultivada, incorporación de la población activa y aportes nutricionales (González et al., 2012). En Venezuela, el arroz constituye uno de los cereales más importantes en la dieta de los venezolanos y su cultivo es de gran importancia económica en las principales zonas agrícolas del país.

La familia Delphacidae o los Delfácidos constituyen un grupo de insectos plagas que producen estragos importantes a nivel mundial en cultivos como arroz, maíz, trigo y sorgo. Estas pérdidas son ocasionadas durante la puesta de huevos, alimentación o transmisión de diferentes fitopatógenos (Remes, 2001; Vivas \& Astudillo, 2008). Tagosodes orizicolus Muir pertenece a esta familia de insectos, la cual puede ocasionar dos tipos de daño, uno proveniente del insecto, llamado daño directo o mecánico y otro por ser trasmisor del virus de la hoja blanca del arroz (VHB) (Vivas \& Astudillo, 2008).

Actualmente, el mejoramiento genético del cultivo ha estado orientado principalmente hacia la obtención de cultivares tolerantes al daño mecánico producido por la sogata, así como el estudio de los mecanismos de resistencia asociados. En relación a esto, Picca, Helguera, Salomón, \& Carrera (2004) mencionan 
que la resistencia en plantas hospederas ha sido clasificada en términos de características anatómicas, morfológicas, fisiológicas y bioquímicas, las cuales son responsables de su expresión, epidemiología, modo de herencia y durabilidad.

Estudios realizados sobre la relación existente entre la anatomía y la producción vegetal demuestran que las condiciones ambientales y nutricionales, así como el estrés a que son sometidas las plantas por efecto de ataque de insectos y/o microorganismos afectan las características estructurales de los vegetales (Sherwood, 1996). Entre esos mecanismos se pueden mencionar: tamaño de la hoja, presencia de tricomas, forma y tamaño de tricomas, acumulación de metabolitos secundarios, organización de las células epidérmicas, tipos de estomas, presencia de cristales de oxalato de calcio y compuestos silíceos, entre muchas otras (Silva et al., 2005).

En las Poaceas, el tejido epidérmico presenta características particulares que han sido utilizadas con fines taxonómicos; sin embargo, muchas de estas estructuras celulares tienen función de protección contra los insectos herbívoros, entre los que se pueden mencionar: presencia de papilas, micropelos y cerdas o aguijones (Ellis, 1986). Diferentes autores coinciden que existen mecanismos que dificultan la digestibilidad por parte de algunos microorganismos y la oviposición de los insectos, los mismos están asociados con el grosor de la cutícula y con la presencia y tipo de tricomas (Lovinger Liewehr \& Lamp, 2000; Molina-Montenegro, Ávila, Hurtado, Valdivia \& Gianoli, 2006).

Entre las teorías que se han propuesto en defensa a los depredadores herbívoros, existe una que abarca tres aspectos importantes como son la protección mecánica en la superficie de la planta, la presencia de cuerpos de sílice y la presencia de tóxicos vegetales que matan o repelen a los insectos. Los compuestos silíceos generalmente inhiben la digestión debido a que algunos animales no poseen las enzimas que permiten degradarlos. Los cuerpos silíceos presentan diferentes localizaciones, los cuales se pueden presentar como frágiles revestimientos de las paredes celulares, realizando sustituciones de la pared celular por ganchos, espinas y tricomas silicificados. Adicionalmente se ha demostrado que la presencia de cuerpos silíceos incrementa la estabilidad mecánica de los tejidos y suministra protección contra microorganismos y depredadores herbívoros en especies de poaceas (Granado-Sánchez, Ruiz-Puga \& Barrera-Escorcia, 2008; Herrera-Giraldo et al., 2009).

En relación al tamaño y forma de los tricomas, Levin (1973) cita que este criterio ha sido usado principalmente con propósitos taxonómicos; sin embargo, el papel principal de ellos está asociado a la defensa contra insectos fitófagos.

Se ha señalado que la caracterización anatómica de la hoja es de gran importancia para los estudios básicos de ecología y físiología, y los mismos son aplicados en las áreas de fitopatología y mejoramiento de plantas (Granado-Sánchez et al., 2008). Por lo anteriormente señalado, es muy importante considerar las características morfoanatómicas de las plantas de arroz resistentes y/o tolerantes al daño mecánico producido por sogata y poder así establecer la relación con los diferentes mecanismos de resistencia registrados, los cuales pudieran ser utilizados en los distintos programas de mejoramiento conducentes hacia la resistencia al insecto.

\section{MATERIALES Y MÉTODOS}

Se realizó un ensayo de resistencia al daño mecánico y mecanismo de resistencia al ataque de sogata, antixenosis y antibiosis para la oviposición, en los Invernaderos de la Fundación Danac-San Javier, estado Yaracuy $\left(10^{\circ} 21^{\prime} 50^{\prime}\right.$ N - 6859'15'W), Venezuela (Cuadro 1). De estos ensayos, se seleccionaron muestras de tejido foliar de los seis cultivares después de ocho días de infestación con los insectos provenientes de una colonia sana (Velásquez, 2013). Para los análisis histológicos, se seleccionó al azar cinco plantas y de ellas se muestreó el tercio medio de la tercera hoja para el montaje 
CUADRO 1

Cultivares de arroz y mecanismo de resistencia al daño mecánico del insecto sogata

\begin{tabular}{lcc}
\multicolumn{1}{c}{ Cultivares } & \multicolumn{2}{c}{ Mecanismo de resistencia } \\
Makalioka $(+)$ & Testigo de Resistencia (Antixenosis y Antibiosis) \\
D-Sativa $(*)$ & Antixenosis & Antibiosis intermedio \\
Venezuela $21(*)$ & Preferencia intermedia & Susceptible \\
Cimarrón $(*)$ & \\
Zeta $15(*)$ & \multicolumn{2}{c}{ Testigo de Susceptibilidad } \\
Bluebonnet $50(-)$ & \\
\hline
\end{tabular}

(*) Cultivar venezolano; (+) Testigo resistente, (-) Testigo susceptible. Fuente: Velásquez (2013).

microscópico (Rodríguez, Negrín \& García, 2009; Ramiro, Oliveiro-Guerrero, Quiroz-Vol$\tan \&$ Chebabi-Matthiesen, 2004). Las muestras fueron fijadas en una solución de FAA 70\% hasta su procesamiento en el Laboratorio de Anatomía Vegetal del Centro de Investigaciones en Biotecnología Agrícola (CIBA-IGen) de la UCV-Maracay, Estado Aragua $\left(10^{\circ} 16^{\prime} 12^{\prime \prime} \mathrm{N}\right.$ - 67³6’43”W). El estudio de las epidermis en vista paradérmica se realizó luego de someter el material vegetal a un proceso de macerado parcial con una solución de agua destilada e hipoclorito de sodio al 2.5\% (V:V), la tinción se realizó con azul de toluidina acuosa $(0.5 \%)$ y el montaje se hizo en una solución glicerina-agua (V:V), preparándose láminas semipermanentes (Roth, 1964). Los cortes de las secciones transversales fueron realizados a mano alzada, y teñidos con azul de toluidina acuosa al 1\%. Las observaciones fueron realizadas en un microscopio marca Nikon Eclipse E200, equipado con una cámara digital Pixelink Capture SC.

Se evaluaron las características anatómicas asociadas con la resistencia al daño mecánico producido por el insecto, entre ellas: grosor de la cutícula más la pared celular externa, presencia de macro y micropelos, y presencia de cuerpos silíceos (Howe \& Schaller, 2008); Las observaciones fueron realizadas sobre la epidermis adaxial y abaxial de la lámina foliar de los cultivares de arroz seleccionados. El grosor de la cutícula fue medido en el área comprendida entre el haz vascular de la nervadura principal y el haz vascular siguiente. Se utilizó un diseño completamente al azar con cuatro repeticiones, cada repetición estuvo constituida por cinco láminas con cinco secciones histológicas cada una, y se realizaron cinco observaciones por lámina.

Se efectuó un análisis de varianza (ANDEVA) para aquellas características que cumplieron con los supuestos propuestos para la estadística paramétrica, sobre todo para el caso de la normalidad. La diferencia entre medias se evaluó a través de la prueba de Duncan al 5\%. En el caso de las variables que no cumplieron con los supuestos del ANDEVA, el análisis se realizó vía no paramétrica, y se empleó la prueba de Kruskal-Wallis. Se empleó el paquete estadístico InfoStat (InfoStat, 2004).

\section{RESULTADOS}

De la evaluación en el comportamiento entre los cultivares y las variables estudiadas, se observaron diferencias significativas para las variables grosor de la cutícula $(\mu \mathrm{m})$, número de micropelos $/ \mathrm{mm}^{2}$ y longitud de los macropelos $(\mu \mathrm{m})$. Al emplear la prueba de medias de Duncan para el grosor de la cutícula de la cara adaxial (Cuadro 2), se encontraron tres grupos diferenciados. Un primer grupo, formado por el parental BB50, el cual presentó el valor más bajo de grosor de la cutícula $(1.96 \mu \mathrm{m})$, esto demuestra la facilidad que presenta el insecto para alimentarse, y colocar sus posturas sobre el tejido foliar en este cultivar. Un segundo grupo, constituido por Zeta 15 y Makalioka, las cuales presentaron un valor medio de grosor de la cutícula $(2.34 \mu \mathrm{m})$ al compararlo con la media 
CUADRO 2

Valores promedios de las variables grosor de la cutícula, número de micropelos y longitud del macropelo en la cara adaxial y abaxial de la lámina foliar de los cultivares

\begin{tabular}{lcccccc}
\multirow{2}{*}{ Cultivares } & \multicolumn{2}{c}{ Grosor de la cutícula $(\mu \mathrm{m})$} & \multicolumn{2}{c}{ Número de micropelos $/ \mathrm{mm}^{2}$} & \multicolumn{2}{c}{ Longitud de los macropelos $(\mu \mathrm{m})$} \\
& Adaxial & Abaxial & Adaxial & Abaxial & Adaxial & Abaxial \\
Makalioka & $2.34 \mathrm{~b}$ & $2.38 \mathrm{~b}$ & $66.89 \mathrm{bc}$ & $64.62 \mathrm{c}$ & $61.07 \mathrm{a}$ & $52.45 \mathrm{a}$ \\
Cimarrón & $3.05 \mathrm{c}$ & $2.99 \mathrm{~d}$ & $54.97 \mathrm{~b}$ & $30.57 \mathrm{~b}$ & $72.95 \mathrm{a}$ & $85.38 \mathrm{a}$ \\
Venezuela 21 & $2.74 \mathrm{c}$ & $2.57 \mathrm{bc}$ & $88.35 \mathrm{c}$ & $100.02 \mathrm{~d}$ & $149.83 \mathrm{a}$ & $90.26 \mathrm{a}$ \\
Zeta 15 & $2.34 \mathrm{~b}$ & $2.01 \mathrm{a}$ & $110.75 \mathrm{~d}$ & $98.52 \mathrm{~d}$ & $226.20 \mathrm{ab}$ & $169.69 \mathrm{ab}$ \\
D-Sativa & $3.07 \mathrm{c}$ & $2.75 \mathrm{~cd}$ & $71.42 \mathrm{bc}$ & $78.40 \mathrm{c}$ & $381.54 \mathrm{~b}$ & $301.97 \mathrm{c}$ \\
BB50 & $1.96 \mathrm{a}$ & $1.95 \mathrm{a}$ & $11.72 \mathrm{a}$ & $0.00 \mathrm{a}$ & $81.80 \mathrm{a}$ & $226.76 \mathrm{bc}$ \\
\hline
\end{tabular}

Prueba de Duncan: Letras distintas indican diferencias significativas $(\mathrm{p}<=0.05)$.

observada en el grupo tres constituido por los cultivares Venezuela-21, Cimarrón y D-Sativa y cuyos valores oscilaron entre 2.74 y $3.07 \mu \mathrm{m}$.

Para la cara abaxial se observó la formación de seis grupos, donde en el primer grupo se encontraban el testigo de susceptibilidad BB50 y el cultivar Zeta 15, y a partir del segundo grupo se evidenció un mayor grosor de la cutícula, diferenciándose del testigo de resistencia Makalioka (Cuadro 2), ante estos resultados se pudiera inferir que este carácter pudiera estar asociado a una diferencia taxonómica. En la figura 1 se pueden visualizar las diferencias en grosor de los cultivares venezolanos en comparación con los testigos de resistencia y susceptibilidad (Makalioka y Bluebonnet 50 respectivamente).

La correlación del grosor de la cutícula entre ambas caras fue altamente significativa $(p=0.0001)$, demostrando que los insectos pueden posarse indistintamente sobre la cara adaxial y abaxial, como fue observado durante la ejecución del ensayo (Cuadro 3).

Las diferencias altamente significativas observadas entre los cultivares para la variable número de micropelos $/ \mathrm{mm}^{2}$ en la cara adaxial

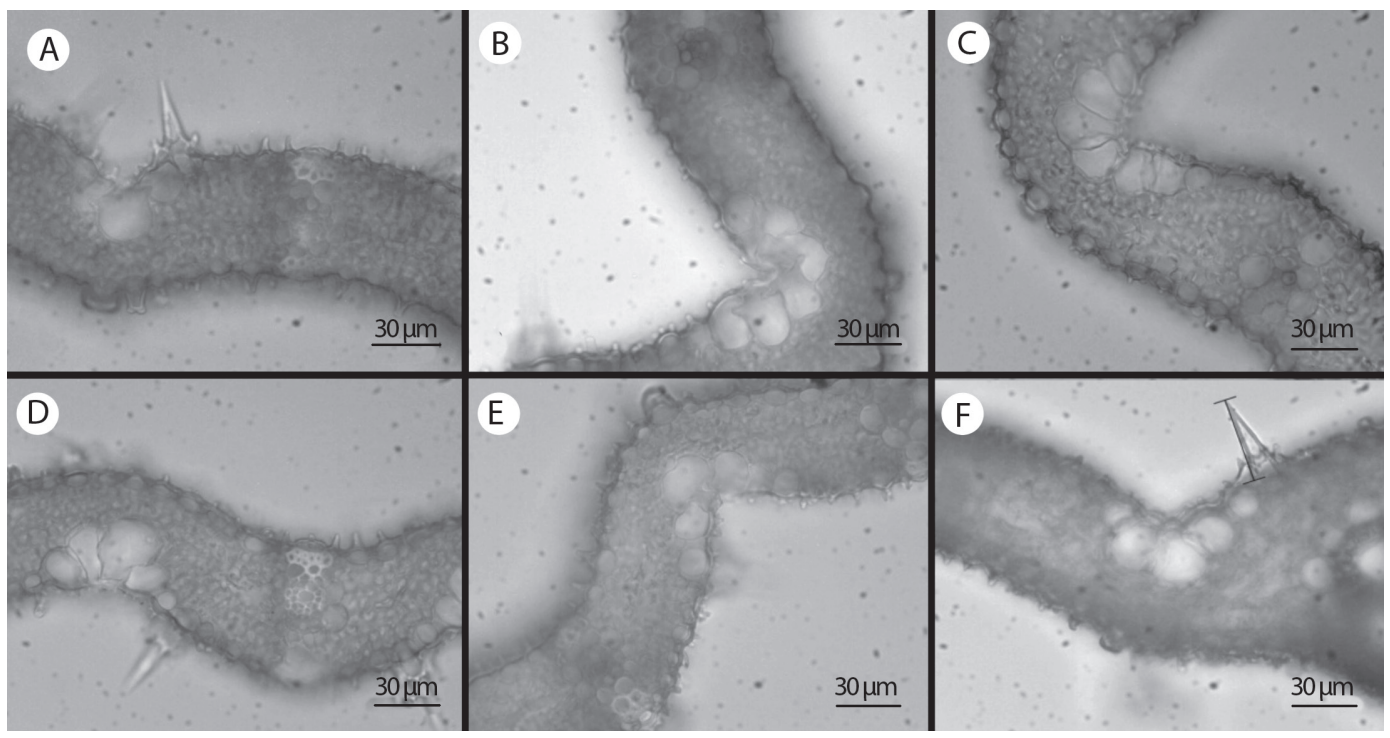

Fig. 1. Grosor de la cutícula más pared celular externa en la cara abaxial de los tipos de arroz evaluados: A. Cimarrón, B.

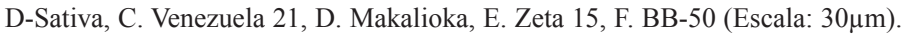


CUADRO 3

Correlación entre las variables morfo anatómicas, número de huevos de sogata (NHuevos) y número de insectos posados a las $96 \mathrm{~h}(\mathrm{p}<0.05)$

\begin{tabular}{|c|c|c|c|}
\hline Variable 1 & Variable 2 & Correlación & Probabilidad \\
\hline GroAbx & $96 \mathrm{~h}$ & -0.5940 & $0.0022 *$ \\
\hline GroAbx & GroAdx & 0.7760 & $<.0001 *$ \\
\hline longmad & $96 \mathrm{~h}$ & 0.1536 & 0.4736 \\
\hline longmad & NHuevos & -0.1173 & 0.5850 \\
\hline longmad & GroAdx & 0.3152 & 0.1335 \\
\hline longmad & GroAbx & -0.0603 & 0.7795 \\
\hline longmad & $\operatorname{micadx}$ & 0.3582 & 0.0857 \\
\hline longmad & macad & -0.2354 & 0.2682 \\
\hline $\mathrm{mic} / \mathrm{mm} 2 \mathrm{ab}$ & $\operatorname{micadx}$ & 0.8636 & $<.0001 *$ \\
\hline $\mathrm{mic} / \mathrm{mm} 2 \mathrm{ab}$ & macad & -0.3980 & 0.0541 \\
\hline $\mathrm{mic} / \mathrm{mm} 2 \mathrm{ab}$ & longmad & 0.4021 & 0.0514 \\
\hline longmab & $96 \mathrm{~h}$ & 0.1425 & 0.5064 \\
\hline longmab & NHuevos & -0.0316 & 0.8834 \\
\hline longmab & GroAdx & 0.0896 & 0.6771 \\
\hline longmab & GroAbx & -0.2287 & 0.2824 \\
\hline longmab & $\operatorname{micadx}$ & -0.1455 & 0.4975 \\
\hline longmab & macad & 0.1545 & 0.4711 \\
\hline longmab & longmad & 0.4881 & $0.0155^{*}$ \\
\hline longmab & $\mathrm{mic} / \mathrm{mm} 2 \mathrm{ab}$ & -0.0142 & 0.9474 \\
\hline longmab & macab & -0.2502 & 0.2383 \\
\hline sílice & $96 \mathrm{~h}$ & -0.3244 & 0.1220 \\
\hline sílice & NHuevos & -0.1376 & 0.5215 \\
\hline sílice & GroAdx & 0.3822 & 0.0653 \\
\hline sílice & GroAbx & 0.2379 & 0.2631 \\
\hline sílice & $\operatorname{micadx}$ & 0.6612 & $0.0004 *$ \\
\hline sílice & macad & -0.4739 & $0.0193 *$ \\
\hline sílice & longmad & 0.2637 & 0.2131 \\
\hline sílice & $\mathrm{mic} / \mathrm{mm} 2 \mathrm{ab}$ & 0.6198 & $0.0012 *$ \\
\hline sílice & macab & -0.1527 & 0.4764 \\
\hline sílice & longmab & -0.2258 & 0.2888 \\
\hline
\end{tabular}

GroAbx: grosor cara abaxial; GroAdx: grosor cara adaxial; longmad; longitud macropelo cara adaxial; longmab: longitud macropelo cara abaxial; macad: macropelos cara adaxial; macab: macropelos cara abaxial; micadx: micropelos cara adaxial; micabx: micropelos cara abaxial; NHuevos: número de huevos; 96h: insectos posados a las 96h; sílice: cuerpos silíceos.

y abaxial del tejido foliar, demuestran la asociación que existe entre esta variable y el nivel de resistencia al daño mecánico producido por sogata, mientras que la prueba de medias de Duncan para el número de micropelos $/ \mathrm{mm}^{2}$ presentes en la cara adaxial y abaxial, arrojó la formación de cinco y cuatro grupos bien diferenciados respectivamente (Cuadro 2), donde el cultivar BB50 presentó la menor cantidad de micropelos en ambas caras y Makalioka con valores intermedios para el número de micropelos presentes $\left(66.89 \mathrm{micropelos} / \mathrm{mm}^{2}\right.$ cara adaxial y $64.62 \mathrm{micropelos} / \mathrm{mm}^{2}$ cara abaxial). La tendencia de los otros cultivares para formar grupos de medias, fue similar en ambas epidermis, Cimarrón se ubicó en el grupo dos para ambas epidermis con un número promedio de 54.97 micropelos $/ \mathrm{mm}^{2}$ en la cara adaxial y $30.57 \mathrm{micropelos} / \mathrm{mm}^{2}$ en la abaxial. D-Sativa comparte el grupo tres con Makalioka con 
valores intermedios para ambas caras, mientras que Venezuela-21 y Zeta 15 se ubicaron en dos grupos diferentes para la cara adaxial y en un mismo grupo para la cara abaxial, con los mayores números de micropelos por unidad de área. Estos resultados indican, que los genotipos que presentaron un número de micropelos por unidad de área superior a la observada en Makalioka (66.89 en la cara adaxial o 64.62 en la abaxial) presentan una mayor resistencia al ataque del insecto, afectando así su comportamiento para la oviposición.

Para el caso, de la longitud de los macropelos se observaron diferencias significativas en ambas epidermis en cada uno de los materiales evaluados, donde para la cara adaxial (Cuadro 2) se pudo evidenciar la presencia de tres grupos bien diferenciados. Un primer grupo donde se encontraba tanto los testigos, Makalioka y BB50, como también Cimarrón y Venezuela-21. Un segundo grupo formado por Zeta $15 \mathrm{y}$ un tercer grupo constituido por el cultivar D-Sativa. Estos resultados indican que la variable longitud del macropelo en la cara adaxial no es un carácter significativo para medir el grado de resistencia o susceptibilidad al daño mecánico producido por el insecto en arroz. Por otro lado, en la cara abaxial, se observaron diferencias significativas entre los cultivares para la longitud de los macropelos, y se formaron cuatro grupos durante la comparación de medias. Un primer grupo donde estuvieron ubicados Makalioka, Cimarrón y Venezuela-21, con similar comportamiento, con valores comprendidos entre 52.45 y $90.26 \mu \mathrm{m}$. Un segundo grupo formado por Zeta 15 con una longitud de $169.9 \mu \mathrm{m}$, el cultivar BB50 se ubicó en el tercer grupo con una longitud promedio de $226.75 \mu \mathrm{m}$ y el último grupo constituido por D-Sativa con una longitud de $301.97 \mu \mathrm{m}$, presentando además los tricomas más largos (Cuadro 2). Para el número de macropelos no se encontraron diferencias entre los cultivares en ambas caras.

El análisis no paramétrico para la variable presencia de cuerpos silíceos, mostró diferencias altamente significativas $(\mathrm{p}<0.0001)$ entre los cultivares evaluados, lo que demuestra que estos compuestos químicos interfieren con el comportamiento del insecto en los cultivares de arroz evaluados. Se detectó una correlación positiva significativa entre los cuerpos silíceos y la presencia de micropelos y macropelos en la cara adaxial y abaxial (Cuadro 3).

Al realizar la prueba de medianas de Kruskall-Wallis se encontró la formación de dos grupos, donde un grupo estuvo conformado por BB-50, y el otro grupo por el resto de los cultivares incluyendo Makalioka, esto explica porque la mayoría de los materiales aquí evaluados presentaron cierto grado de resistencia al daño mecánico ocasionado por el insecto y que el mismo está asociado con la presencia de cuerpos silíceos en los espacios intercostales. En la figura 2, se muestra las hileras de sílice presentes en el tejido epidérmico de los materiales evaluados, a pesar de no existir diferencias entre los cultivares y el testigo de resistencia al daño mecánico, se puede inferir que mientras mayor es la cantidad de sílice presente en el tejido epidérmico mayor será la

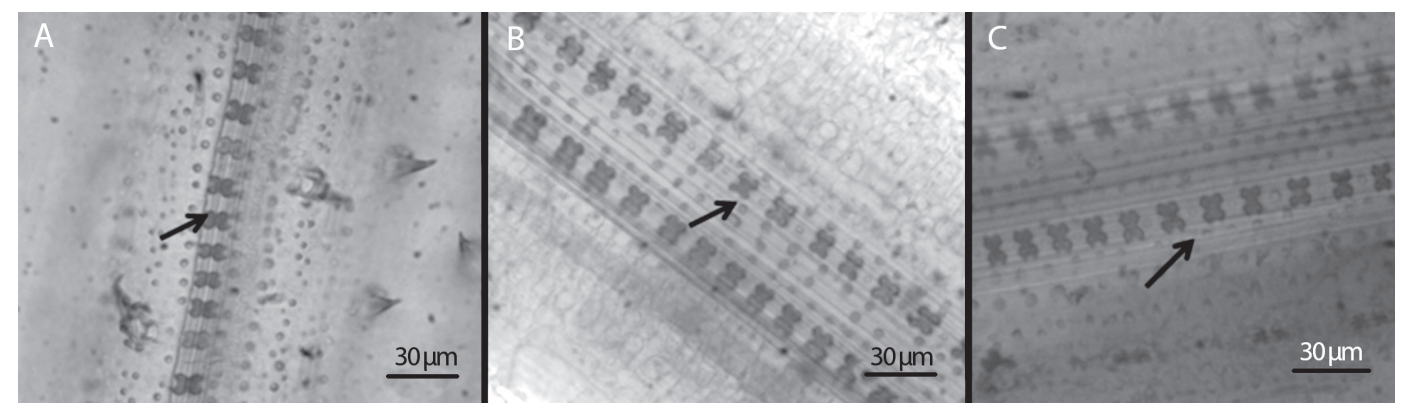

Fig. 2. Presencia de cuerpos silíceos (flecha) en el tejido foliar de los tipos de arroz: A. Venezuela-21; B. Makalioka; C. D-Sativa $(30 \mu \mathrm{m})$. 
resistencia al ataque del insecto. La presencia de cuerpos silíceos estuvo correlacionada significativamente de forma positiva con la presencia de micropelos en el tejido epidérmico y negativamente con la presencia de macropelos en la cara adaxial (Cuadro 3).

\section{DISCUSION}

Las diferencias significativas encontradas para el grosor de la cutícula en ambas caras del tejido epidérmico coincide con los resultados registrados por Rodríguez et al. (2009) y Ramiro et al. (2004) quienes indican que el grosor de la cutícula es una de las características que afecta el comportamiento de los insectos herbívoros para alimentarse y ovipositar. Los resultados encontrados demuestran la asociación que existe entre la variable grosor de la cutícula y los mecanismos de resistencia, antixenosis y antibiosis para la oviposición, al ataque de sogata encontrado en los cultivares de arroz en Venezuela. Resultados similares son citados por Melo, Kraus \& Menezes. (2002) al realizar estudios anatómicos del tejido foliar en plantas de Asteraceae, determinaron que la presencia de una cutícula más gruesa en la cara adaxial impide el ataque de larvas de lepidópteros.

En Poaceas, la presencia de micropelos ha sido utilizada con fines taxonómicos como lo menciona Ellis (1979), sin embargo, en esta investigación se demostró que estos caracteres morfoanatómicos están asociados con la no preferencia del insecto sogata a posarse sobre las hojas de arroz, coincidiendo con lo señalado por Howe \& Schaller (2008) al indicar que la presencia de micropelos y aguijones en el tejido foliar de las Poaceas influyen positivamente en el desarrollo de un mecanismo de resistencia al ataque de insectos herbívoros. Igualmente, Singh \& Singh (2005) señalan que el ataque de Empoasca spp. en algodón estuvo asociado de manera consistente al número de pelos presentes en el tejido foliar, a mayor número de pelos mayor fue la resistencia al ataque del insecto, evitando que el insecto se pose sobre las hojas produciendo un daño mecánico.
Por otro lado, Showler (2009) refirió la presencia de pelos asociados como un mecanismo de no preferencia al ataque del picudo del algodón (Anthonomus grandis). De la misma manera, Eickhoff, Heng-Moss, Baxendale \& Foster (2008) indican que la presencia de micropelos en el tejido foliar de los cultivares de pasto búfalo resistentes a la chinche (Blissus occiduus Barber) era debida a un mecanismos de antixenosis que afectan el comportamiento del insecto.

Calatayud \& Munera (2000) reportaron que el mecanismo de resistencia en yuca al ataque de trips, era debida a la alta pilosidad encontrada en las hojas de las plantas resistentes, ya que estas afectaron el establecimiento y la alimentación sostenida de la plaga en la planta. Sin embargo, Pathak (1977) indicó que aunque la vellosidad en la hoja bandera podría actuar como una barrera física a la postura de huevos del barrenador en arroz (Chilo suppressalis Wlk), la no presencia o poca cantidad de macropelos observados en la cultivar resistente TKM-6, no la hace menos atractiva para que el insecto coloque sus huevos, y esto está asociado con el desarrollo de nuevos biotipos de insectos.

La longitud del macropelo pareciera no estar asociada con ningún mecanismo de resistencia, ya que estos macropelos son unicelulares, no secretores, tal como lo clasifica Ellis (1986), y los mismos han sido utilizados con fines taxonómicos dentro de las Poaceas. Sin embargo, esta característica impide que los insectos se posen con facilidad sobre la epidermis de las plantas resistentes dificultando su alimentación y colocación de huevos.

En relación a la presencia de los cuerpos silíceos, Herrera-Giraldo et al. (2009) registraron que las células de sílice y las de corcho presente en las células cortas del tejido epidérmico de plantas de arroz están llenas de $\mathrm{SiO}_{2}$, este compuesto ha sido considerado por algunos autores como el principal mecanismo de defensa de las plantas a los insectos herbívoros, porque reduce la digestibilidad y da mayor dureza y abrasividad al tejido (Granado-Sánchez et al. 2008; Massey, Ennos \& Hartley, 2006). 
Por otro lado, Molina-Montenegro et al. (2006) y Howe \& Hander (2008) al trabajar con algunas especies de Poaceas indican que la presencia de cuerpos silíceos, asociados a un gran número de estructuras anatómicas (micropelos, papilas y aguijones) en el tejido epidérmico de la hoja, juega un papel importante al momento de seleccionar cultivares resistentes al ataque de insectos fitófagos, resultados similares son señalados por Ellis (1979) al indicar que el mayor contenido de sílice se encuentra presente en el tejido intercostal (micropelos y aguijones) de la lamina foliar y en mayor proporción en la cara abaxial, demostrando que la resistencia al daño mecánico producido por sogata en los cultivares de arroz venezolano está asociado principalmente con el contenido de sílice presente en las células epidérmicas.

A pesar que no se encontró diferencias estadísticas para el numero de macropelos presentes en el tejido foliar, se observó una correlación significativa entre el número de macropelos en la cara abaxial y los insectos posados a las 96h (Cuadro 3), que coincide con lo citado por Cardozo, Cristiano, Morales \& Martins (2009) y Pathak (1977) al indicar que la antixenosis es producto de la acción de caracteres físicos presentes en el tejido foliar.

Finalmente se puede concluir que bajo las condiciones de invernadero en que fue realizada esta investigación, el grosor de la cutícula es una de las características morfoanatómicas de mayor relevancia al estudiar la asociación con el mecanismo de resistencia al daño mecánico producido por sogata en arroz, porque esta variable constituye una barrera física que impide que el insecto se alimente y ovoposite sobre la planta. Igualmente se determinó que la presencia de micropelos y macropelos en el tejido epidérmico esta asociado con el grado de resistencia al ataque del insecto, producto del contenido de cuerpos silíceos en los espacios intercostales.

\section{AGRADECIMIENTOS}

Al Consejo de Desarrollo Científico y Humanístico de la Universidad Central de
Venezuela (CDCH-UCV) por el financiamiento del proyecto de Investigación.

\section{RESUMEN}

El arroz es afectado directa e indirectamente por el insecto sogata. El daño directo o mecánico es debido a la postura de huevos y alimentación del tejido foliar por parte del insecto y el daño indirecto que ocurre es por la trasmisión del virus de la hoja blanca del arroz. El ensayo fue conducido en la Fundación DANAC en agosto 2011, con la finalidad de evaluar las estructuras morfoanatómicas asociadas con la resistencia al daño mecánico en seis cultivares de arroz que incluyen a los testigos de resistencia y susceptibilidad (Bluebonnet 50 y Makalioka). En el tercio medio de la tercera hoja, se evaluaron las variables grosor de la cutícula, presencia de macropelos, micropelos y cuerpos silíceos. Se observaron diferencias significativas entre los cultivares para el grosor de cutícula, número de micropelos y presencia de cuerpos silíceos, se determinó una correlación significativa entre el número de macropelos y micropelos en la cara adaxial con la presencia de cuerpos silíceos y el grosor de la cutícula y el número de insectos posados a las $96 \mathrm{~h}$. El grosor de la cutícula y la presencia de cuerpos silíceos en los espacios intercostales de los micropelos y macropelos mostraron ser los caracteres más relacionados con la resistencia al daño mecánico y los mecanismos de resistencia al insecto.

Palabras clave: arroz, sogata, resistencia, anatomía, mecanismos de resistencia.

\section{REFERENCIAS}

Cardozo, D., Cristiano, M., Morales, L., \& Martins, T. (2009). Does thricomes on plant epidermis surface disturb ant locomotion? American Journal of Agriculture and Biological Science, 4(1), 1-6.

Calatayud, P. \& Munera, D. (2000). Defensa Natural de la yuca a las plagas artrópodos. Memorias XXVIII Congreso SOCULEN, Cali, Colombia.

Eickhoff, T., Heng-Moss, T., Baxendale, F., \& Foster, J. (2008). Levels of Tolerance, Antibiosis, and Antixenosis among Resistant Buffalo grasses and Zoysia grasses. Journal of Economic Entomology, 101(2), 533-540.

Ellis, R. P. (1979). A procedure for standardizing comparative leaf anatomy in the Poaceae II. The epidermis as seen in surface view. Bothalia, 12, 641-647.

Ellis, R. P. (1986). A review comparative leaf blade anatomy in the Systematics of the Poaceae: The past twenty-five years. In T. R. Soderstrom, K. Hilu, C. Campbell, \& M. Barkworth (Eds.). Grass Systematics 
and Evolution (pp. 3-10). Washington, D C.: Smithsonian Institute.

Granado-Sánchez, D., Ruiz-Puga, P., \& Barrera-Escorcia, H. (2008). Ecología de la herviboría. Revista Chapingo serie Ciencias Forestales y de Ambiente, 14(1), 51-63.

González-Vera, A., Labrín, N., Álvarez, R. M., Jayaro, Y., Gamboa, C., Barrientos, V., \& Reyes, E. (2012). Mechanisms of Oryza sativa (Poaceae) resistance to Tagosodes orizicolus (Homoptera: Delphacidae) under greenhouse condition in Venezuela. Revista de Biología Tropical, 60(1), 105-117.

Herrera-Giraldo, C., Gómez-Barrera, M., Saavedra-Hernández, M., Fonthal-Rivera, G., Gonzalez-Ceballos, V., \& Calderón, H. (2009). Cuantificación de sílice presente en el follaje de Guadua angustifolia del departamento del Quindío. Revista Investigación de Universidad Colombia, 19, 14-17.

Howe, G. \& Hander, G. (2008). Plant immunity to insect herbivorous. Annual Review of Plant Biology, 59, 41-66.

Howe, G. \& Schaller, A. (2008). Direct defense in plants and their induction by wounding and insect herbivores. In A. Schaller (Ed.), Induce plant resistance to herbivory (pp. 7-29). Alemania: Springer.

InfoStat. (2004). Manual del usuario. Grupo Infostat, FCA, Universidad Nacional de. Córdoba. Argentina: Editorial Brujas.

Levin, D. (1973). The role of trichomes in plant defense. The quarterly Review of Biology, 48(1), 3-15.

Lovinger, A., Liewehr, D., \& Lamp, W. (2000). Glandular trichomes on alfalfa impede searching behavior of the potato leafhopper parasitoid. Biological Control, $18,187-192$.

Massey, F., Ennos, A. \& Hartley, S. (2006). Silica in grasses as a defense against insect herbivorous; contrasting effects on folivorous and a phloem feeder. Journal of Animal Ecology, 75, 595-603.

Melo De Pinna, G. F. A., Kraus, J. E., \& Menezes, N. L. (2002). Morphology and Anatomy of Leaf Mine in RichteragoRiparia Roque (Asteraceae) in the Campos Rupestres of Serra Do Cipó, Brazil. Brazilian Journal Biology, 62(1), 179-185.

Molina-Montenegro, M. A., Ávila, P., Hurtado, R., Valdivia, A. I., \& Gianoli, E. (2006). Leaf trichome density may explain herbivory patterns of Actinote sp. (Lepidoptera: Acraeidae) on Liabummandonii (Asteraceae) in a montane humid forest (Nor Yungas, Bolivia). Acta Ecológica, 30, 147-150.
Pathak, M. D. (1977). Defense of the Rice Crop Against Insect Pests. Annals of the New York Academy of Sciences, 287(1), 287-295.

Picca, A., Helguera, N., Salomón, A., \& Carrera, A. (2004). Marcadores Moleculares. In V. Echenique, C. Rubinstein, \& L. Mogrisdki (Eds.), Biotecnología y Mejoramiento Genético Vegetal (pp. 125-133). Buenos Aires: Ediciones INTA.

Ramiro, D. A., Oliveiro-Guerrero, F., Quiroz-Voltan, R., \& Chebabi-Matthiesen, S. (2004). Caracterização anatômica de folhas de cafeeiros resistentes e susceptíveis ao bicho-mineiro. Campinas, 63(3), 363-372.

Remes, M. (2001). Tagosodes orizicolus (Muir, 1926) vector del virus de la hoja blanca del arroz (HBV) en la República Argentina (Homoptera-Delphacidae). Revista de la Facultad de Agronomía La Plata, 104(2), 151-156.

Rodríguez, G., Negrín, M., \& García, M. 2009. Evaluación de algunas variables de la epidermis foliar en tres clones de Musa y su relación con resistencia a sigatoka (Mycosphaerella sp.). Revista de Facultad de Agronomía Universidad Central de Venezuela, 35(3), 100-105.

Roth, I. 1964. Microtécnica Vegetal. Caracas: Imprenta Universitaria, Facultad de Ciencias, Universidad Central de Venezuela.

Sherwood, R. T. (1996). Anatomical and physiological mechanisms of resistance to Brown Leaf Spot in smooth bromegrass. Crop Science, 36, 239-242.

Silva, L. M., Alquidini, Y., \& Cavallet,V. J. (2005). Inter-relaçoes entre a anotomía vegetal e a produçao vegetal. Acta Botanical Brasilera, 19(1), 183-194.

Singh D. P. \& Singh, A. (2005). Disease and Insect Resistance in Plants. New Hampshire, USA: Science Publisher.

Showler, A. T. (2009). Roles of Host Plants in Boll Weevil Range Expansion beyond Tropical Mesoamerica. American Entomology, 55(4), 234-242.

Velásquez, S. (2013). Caracterización morfoanatómica, molecular y genética de la resistencia al daño mecánico producido por sogata (Tagosodes orizicolus Muir) en arroz. (Tesis Doctoral). Universidad Central de Venezuela, Facultad de Agronomía, Maracay, Venezuela.

Vivas, C. L. E. \& Astudillo, D. (2008). Enfermedades virales transmitidas por la familia Delphacidae con énfasis en el insecto sogata (Tagosodes orizicolus Muir). INIA Hoy, 1, 1-19. 
